\title{
Atypical Granulomatous Lesion of Gingiva: A Case Report on Rare Manifestation of Tuberculosis in Oral Cavity
}

\author{
Admaja $\mathrm{KN}^{* 1}$ and Thomas $\mathrm{V}^{2}$ \\ ${ }^{1}$ Department of Oral Medicine and Radiology, Mar Baselios Dental College, Kothamangalam, India \\ ${ }^{2}$ Department of Oral Medicine and Radiology, Government Dental College, Thiruvananthapuram, India
}

*Corresponding author: Admaja KN, Department of Oral Medicine and Radiology, Mar Baselios Dental College, Kothamangalam, India, Tel: +91 8129392121, E-mail: admajaknair@gmail.com

Citation: Admaja KN, Thomas V (2017) Atypical Granulomatous Lesion of Gingiva: A Case Report on Rare Manifestation of Tuberculosis in Oral Cavity. J Dent Oral Care Med 3(2): 203. doi: 10.15744/2454-3276.3.203

Received Date: March 23, 2017 Accepted Date: September 19, 2017 Published Date: September 21, 2017

\begin{abstract}
Primary tuberculosis lesion in oral cavity is a rare clinical entity. We report a case of gingival tuberculosis manifested as chronic ulceration with pus discharge. Histopathological examination of the lesion suggested a chronic granulomatous lesion. Mantoux test was strongly positive, but immunological investigations for detection of mycobacterium tuberculosis antibodies were negative. Other oral granulomatous lesions like deep fungal infection, Wegner's granulomatoses etc were ruled out by proper investigations. The lesion as curative on antituberculosis therapy and patient is under follow up. With the decreased incidence of TB, unusual manifestations of oral TB are likely to be missed. The clinician needs to be aware of these various lesions and should consider TB in the differential diagnosis of any nodular, ulcerated lesions of the oral cavity.

Keywords: Atypical tuberculosis; Gingiva; Granulomatous lesion
\end{abstract}

\section{Introduction}

Granulomatous lesion represents a chronic inflammatory reaction with predominance of macrophages and variable amount of lymphocytes [1]. A wide variety of diseases like infectious diseases, sarcoidosis, crohns disease, Wegener's granulomatosis, rheumatoid arthritis, berillyosis, drug reactions or foreign body aspiration may manifest as granulomatous lesions. Among these infective agents like mycobacterium are found to be most commonly associated [2]. Many of these clinical presentations can be uncharacteristic, so that accurate diagnosis and management proves often intractable.

Tuberculosis (TB) is a chronic infectious granulomatous multisystem disease with varied clinical manifestations and is the most common cause of infectious disease-related mortality worldwide. Although effective chemotherapeutic agents are available, TB still accounts for a substantial number of deaths annually with global case-fatality rates are reported to be between $7 \%$ and $35 \%$. Early diagnosis is challenging, even in areas with abundant medical resources. TB is a disease caused usually by Mycobacterium tuberculosis bacteria, transmitted through the respiratory tract by inhaling droplets. The primary form of disease is most often localized to the lung.

Oral manifestation of TB is rare with an incidence of $1.4 \%$ of total TB cases [3]. The primary form of oral TB is rare, occurs preferentially in younger age group and mouth can be the initial site of infection. Secondary orofacial TB arises subsequent to TB from another site and thus may reflect oral inoculation with sputum or haematogenous spread of mycobacteria and usually occurs in all age groups. Floor of the mouth, soft palate, gingiva, lips and hard palate can be involved; however, the tongue tip, dorsum, lateral borders, and base and palate are the most common sites of involvement for oral lesions [4]. We report a case of primary oral TB with two intraoral non-healing ulcers present in a 34-year-old individual without any systemic manifestations.

\section{Case History}

A 34 year old female patient reported with pain \& gradually increasing ulceration of upper gums 1 month duration (Figure 1). No associated symptoms were present. Medical history revealed recurrent episodes of fever during night time, hearing loss and weight loss for the past 6 months. Associated dysphagia and loss of appetite were also present. Patient had habit of mouth breathing. Extra oral examination revealed tender bilateral cervical lymphadenopathy along with pallor. There was conductive deafness in right ear. 
Intra oral examination revealed granular erythematous lesion involving labial \& palatal gingiva extending from 13 to 33 region well defined with ulceration \& pus discharge covered by necrotic slough. On palpation the edges were irregular and undermined with no induration at the base. Clinically differential diagnosis of tuberculous gingivitis, deep fungal infection as well as other granulomatous lesions like Wegener's granulomatosis was considered.

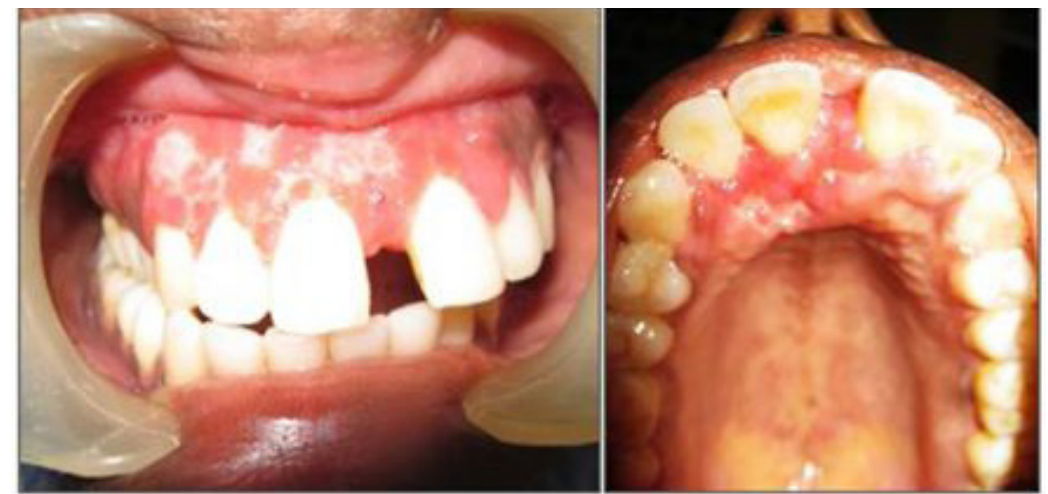

Figure 1: Ulcerative granulomatous lesion on labial and palatal gingiva 13 to 33 region

The radiographic study of 13 to 33 area did not reveal any abnormality. PA Chest radiograph showed evidence of hilar shadows (Figure 2). Routine blood examination showed elevated ESR of $7 \mathrm{O} \mathrm{mm} / \mathrm{hr}$ and Reactive lymphocytosis was noted on peripheral smear evaluation. Histopathological examination revealed pseudoepitheliomatous proliferation, with moderately collagenous connective tissue and infiltration of plasma cells and lymphocytes which was suggestive of a chronic granulomatous inflammation. Focal granulomas were seen, with occasional multinucleated giant cells. Mantoux test was strongly positive (29mm), but sputum AFB, PAS staining as well as TB IgG, IgM were negative. Antineutrophil cytoplasmic antibodies (cANCA and pANCA) test for Wegener's granulomatosis also were negative. Final diagnosis was atypical granulomatous lesion of gingiva.

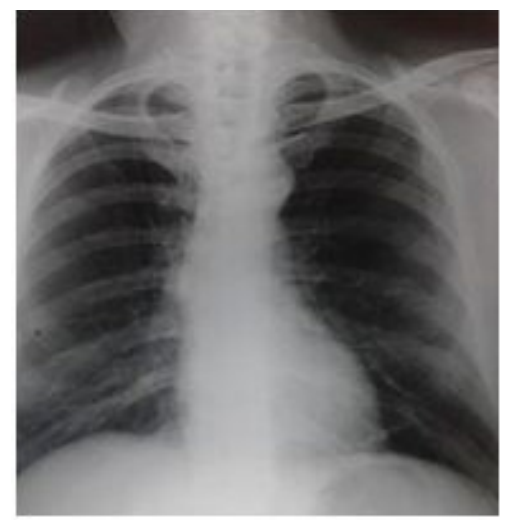

Figure 2: Chest radiograph showing presence of hilar shadows

The patient was initially treated with topical (clotrimazole troche $10 \mathrm{mg}$ ) and systemic antifungals (fluconazole tablet). But there was no clinical remission and PAS staining was also negative ruling out fungal infection. Topical corticosteroids (topical betamethasone $10 \mathrm{mg}$ ) mouth rinse was also non responsive. Considering history, strongly positive Mantoux reaction, hilar shadows in chest radiograph and raised ESR, empirical diagnosis of secondary tuberculosis was given. Antituberculous therapy (ATT) was started and the lesion started subsiding within 1 month. The drug regimen consisted of a combination of isoniazid (300mg), rifampin (60mg), pyrazinamide (500mg), and ethambutol (600 mg) once daily for the initial 2 months, followed by a combination of isoniazid and rifampin for the next 4 months. The patient was completely normal at the end of ATT and no recurrence was evident during a follow up period of 6 months (Figure 3 ).
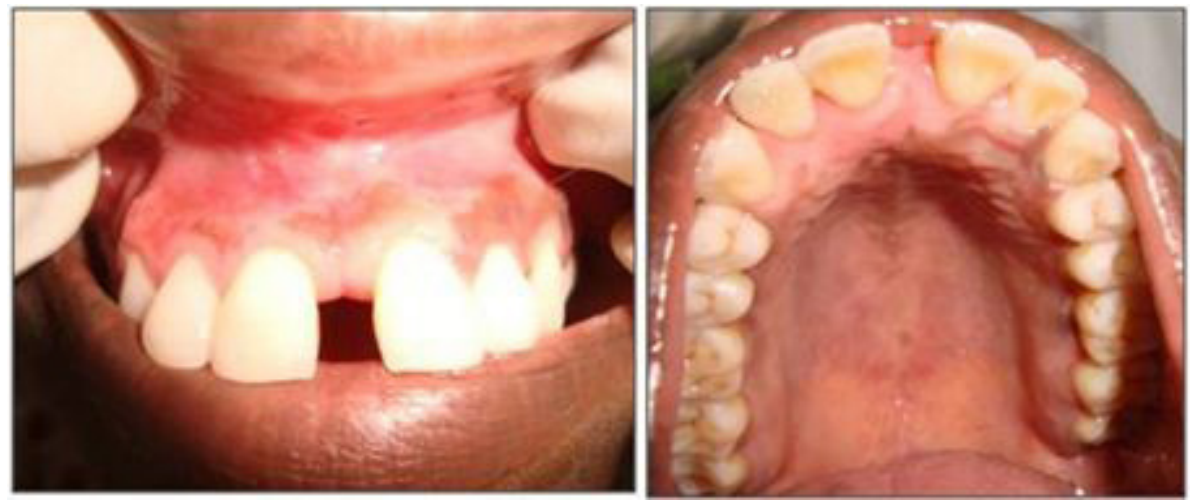

Figure 3: Healed lesion following antituberculous treatment 


\section{Discussion}

Granulomas are inflammatory lesions that evolve in response to irritation, infectious agents, and foreign bodies. Those that are represented by localized masses of fibrovascular granulation tissue accompanied by a nonspecific chronic or sometimes sub-acute inflammatory infiltrate as seen microscopically are simple granulomas; in the oral cavity, lesions of this nature are most frequently encountered intraosseously as endodontically related periapical granulomas [5]. In the oral cavity, specific granulomas are usually associated with mycobacterium or deep invasive fungi, appearing microscopically as casketing and noncaseating granulomas, respectively. The granulomas are charecterized by epithelioid cells and multinucleated giant cells surrounded by $\mathrm{T}$ lymphocytes [6]. Certain types of foreign materials elicit a granulomatous reaction characterized by chronically inflamed granulation tissue and giant cell formation, although not all foreign agents are capable of inducing a giant cell response. Specific infections associated with non casseating granulomatous reactions include mycobacterium avium intercellulare infections, cryptococcosis, histoplasmosis etc. Non caseating granulomas with no identifiable infectious agent may occur as single or multifocal nodules or diffuse swellings in the head and neck area. These lesions constitute an enigmatic group of inflammatory disorders that are subsumed under the rubric of orofacial granulomatosis. Differential diagnosis and management demand a skilful interpretation of clinical findings and pathological evidence [1].

TB is a well-known entity encountered in daily practices. Even though the endemic nature of TB has declined over the past decades, the advent of immuno compromised conditions like HIV impose a significant footing to its prevalence. In such conditions role of atypical mycobacteria has become more relevant.

Classic symptoms are often absent particularly in patients who are immunocompromised or the elderly. Up to $20 \%$ of patients with active TB may be asymptomatic. Classic features associated with active TB are cough, weight loss/anorexia, fever, night sweats, haemoptysis and lymphadenopathy. Signs and symptoms of extrapulmonary TB may be non-specific. They can include leucocytosis, anaemia and hyponatraemia due to the release of antidiurectic hormone release from affected lung tissue [1,5]. All the above classical features were absent in our patient.

"Incipient" and "subclinical" TB are the terms used for characterizing asymptomatic patients with radiographic and/or microbiologic evidence of TB or patients who in retrospect could be defined as having had early states of TB [7].

Cleansing action of saliva, the presence of salivary enzymes, tissue antibodies, oral saprophytes and the thickness of the protective epithelial covering have been proposed as the underlying mechanism attributing to decreased prevalence of oral TB. Any break or loss of these natural barriers, which may be the result of trauma, inflammatory conditions, tooth extraction or poor oral hygiene, may provide a route of entry for the mycobacterium in the primary forms of the disease while for the secondary form the pulmonary bacilli are transmitted to secondary lesions via the lymphatic and haematogenous route [8].

Individuals with characteristic TB symptoms, compatible radiographic abnormalities, and confirmatory mycobacterial cultures have definite TB. In the absence of positive cultures, symptomatic persons with characteristic radiology and/or histopathology are categorized as having culture-negative TB. This diagnosis is confirmed by resolution of abnormalities on antituberculous multidrug therapy. The above mentioned case responded favourably to anti tuberculous therapy eventhough definitive diagnostic tests were negative.

The standard short course treatment for TB is isoniazid, rifampicin, pyrazinamide and ethambutol for 2 months, then isoniazid and rifampicin alone for a further 4 months. The patient is considered cured at 6 months [9]. For latent TB, the standard treatment is 6-9 months of isoniazid alone. If the organism is known to be fully sensitive, then treatment is with isoniazid, rifampicin and pyrazinamide for 2 months, followed by isoniazid and rifampicin for 4 months. Ethambutol need not be used. When situations like resistance to first-line therapy, extensively drug-resistant TB or multidrug-resistant TB arise, the second-line drugs are implemented for the treatment. There are six classes of second-line drugs which include aminoglycosides (amikacin, kanamycin), polypeptides, fluoroquinolones, thioamides (ethionamide, prothionamide), cycloserine and terizidone. A patient with multidrug resistant TB, who remains culture positive after many months of treatment, may be referred for lobectomy or pneumonectomy with the aim of cutting out the infected tissue. Some complications of treated TB like recurrent haemoptysis, destroyed or bronchiectaic lungs and empyema are also amenable to surgical therapy.

\section{Summary}

Atypical presentation of various granulomatous lesions should be considered in the differential diagnosis of any nodular, ulcerated lesions of the oral cavity. The diagnosis and treatment planning in such cases can be a tricky procedure. Conditions like atypical symptomatic TB may be a rarity in the oral cavity, but in such cases empirical treatment modalities can prove beneficial.

\section{References}

1. DG James (2000) A Clinicopathological Classification of Granulomatous Disorders. Postgrad Med J 76: 457-65.

2. Oursler KK, Moore RD, Bishai WR, Harrington SM, Pope DS, et al. (2002) Survival of patients with pulmonary tuberculosis: clinical and molecular epidemiologic factors. Clin Infect Dis 34: 752-9. 
3. Wang WC, Chen JY, Chen YK, Lin LM (2009) Tuberculosis of the head and neck: a review of 20 cases. Oral Surg Oral Med Oral Pathol Oral Radiol Endod 107: 381-6.

4. Sezer B, Zeytinoglu M, Tuncay U, Unal T (2004) Oral mucosal ulceration: a manifestation of previously undiagnosed pulmonary tuberculosis. J Am Dent Assoc 135: 336-40.

5. Eversole LR (2001) Orofacial Granulomatosis and Other Inflammatory Lesions. In Essentials of Oral Medicine 245.

6. Gonzalez-Juarrero M, Turner OC, Turner J, Marietta P, Brooks JV, et al. (2001) Temporal and spatial arrangement of lymphocytes within lung granulomas induced by aerosol infection with Mycobacterium tuberculosis. Infect Immun 69: 1722-8.

7. Oral tuberculosis. eMedicine. April 30, 2007.

8. Achkar JM, Jenny-Avital ER (2011) Incipient and subclinical tuberculosis: defining early disease states in the context of host immune response. J Infect Dis 204: S1179-86.

9. Dadgarnia M, Baradaranfar M, Yazdani N, Kouhi A (2008) Oropharyngeal Tuberculosis: An Unusual Presentation. Acta Medica Iranica 46: 521-4.

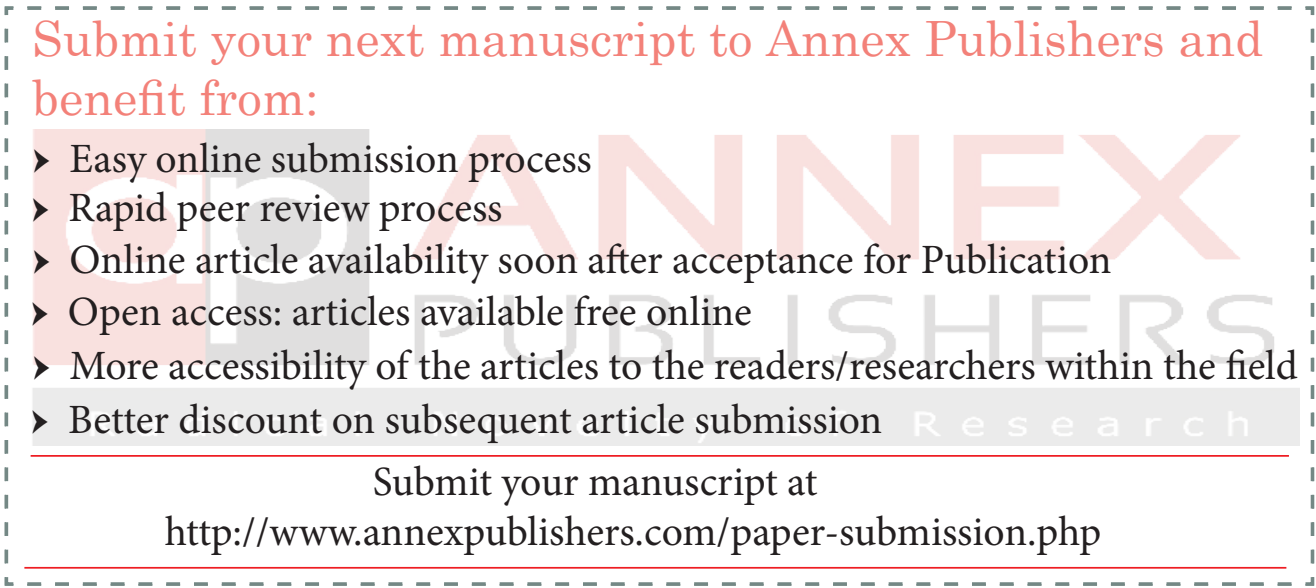

